\title{
Triggered Star Formation from Bubbles S51, N68, and N131
}

\author{
C. P. Zhang and J. J. Wang \\ National Astronomical Observatories, Chinese Academy of Sciences, \\ 100012 Beijing, PR China; email: zcp0507@gmail.com
}

\begin{abstract}
We investigated the environment of the infrared dust bubbles S51 (Zhang \& Wang 2012a), N68 (Zhang \& Wang 2012b), and N131 (Zhang et al. 2012) from catalogue of Churchwell et al. (2006), and searched for evidence of triggered star formation.
\end{abstract}

Keywords. infrared: stars — stars: formation — ISM: bubbles — HII regions
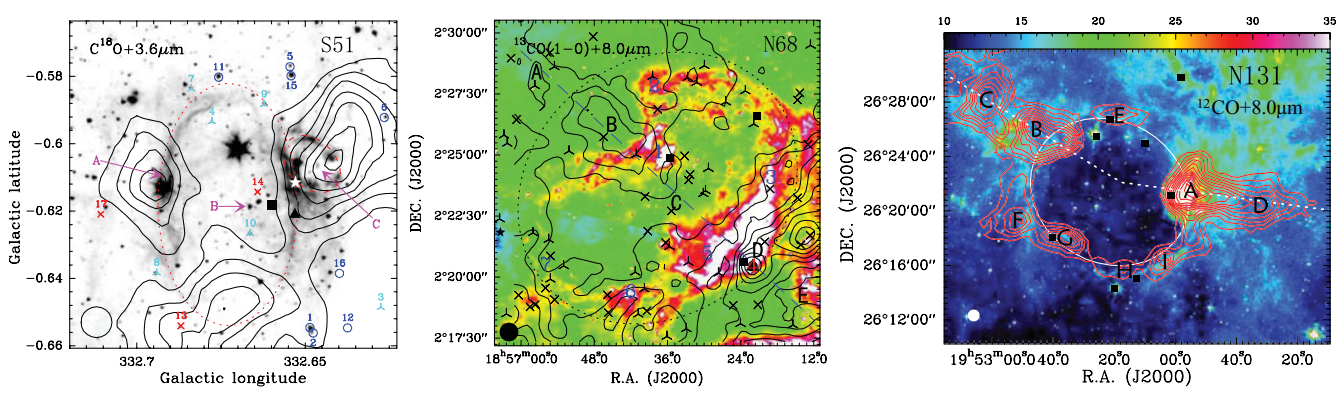

Figure 1. Integrated velocity contours of the $\mathrm{CO}$ emission and young stellar object (YSO) distribution superimposed on the GLIMPSE infrared emission of three bubbles. Detailed information about these three bubbles can be found in Zhang \& Wang 2012a, Zhang \& Wang 2012b, and Zhang et al. 2012.

The ringlike shell of the $\mathrm{CO}$ molecular gas is greatly correlative with the infrared dust emission for the bubbles S51, N68, and N131. The velocity distribution of Mopra ${ }^{13} \mathrm{CO}$ and $\mathrm{C}^{18} \mathrm{O}$ data (Lo et al. 2009) shows that the bubble S51 may be made up of a front-side cloud and a ringlike shell. The position-velocity diagram of GRS ${ }^{13} \mathrm{CO}$ data shows that the bubble N68 may be expanding outward at a speed of about $5 \mathrm{~km} \mathrm{~s}^{-1}$ in the line of sight. Integrated velocity contours and velocity distribution of Delingha ${ }^{12} \mathrm{CO}$ and ${ }^{13} \mathrm{CO}$ data show that there are two giant and collimated wings/flows of $\mathrm{CO}$ emission appearing at the ringlike shell of the bubble N131. Morphologically, it is very obvious that the ringlike shell and the two wings/flows of the bubble N131 were triggered by the exciting star(s) inside this bubble. In addition, several YSOs are located around the ringlike shell of these bubbles, so YSOs may represent a second generation of stars triggered by the bubble's expanding outward.

\section{References}

Churchwell, E., Povich, M. S., Allen, D., et al. 2006, ApJ, 649, 759

Lo, N., Cunningham, M. R., Jones, P. A., et al. 2009, MNRAS, 395, 1021

Zhang, C. P. \& Wang, J. J. 2012a, A\&A, 544, A11

Zhang, C.-P. \& Wang, J.-J. 2012b, arXiv:1208.0423

Zhang, C.-P., Wang, J.-J., \& Xu, J.-L. 2012, A\&\&A, Submitted 